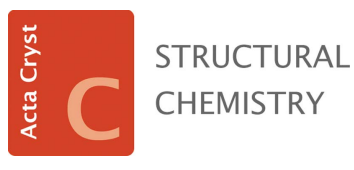

ISSN 2053-2296

Received 12 November 2016

Accepted 4 December 2016

Edited by L. R. Falvello, Universidad de Zaragoza, Spain

Keywords: coproporphyrin I; ruthenium(II) complex; one-dimensional coordination polymer; synchrotron powder diffraction; computational chemistry; molecular cavity.

CCDC reference: 1520660

Supporting information: this article has supporting information at journals.iucr.org/c

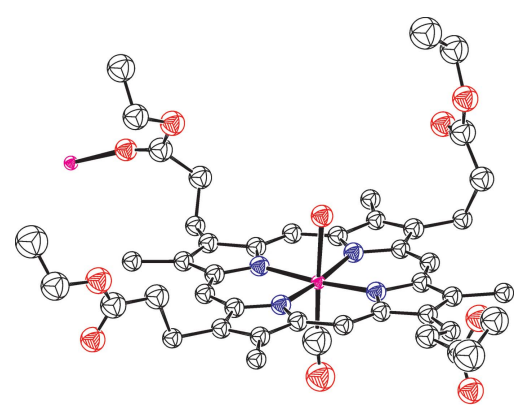

(C) 2017 International Union of Crystallography

\section{Polymeric structure of a coproporphyrin I ruthenium(II) complex: a powder diffraction study}

\author{
Sergey V. Andreev, ${ }^{\text {a,b }}$ Sergey A. Zverev, ${ }^{\text {a,c }}$ Ilya A. Zamilatskov, ${ }^{\text {a,c }}$ Nadezhda M. \\ Kurochkina, ${ }^{a}$ Gelii V. Ponomarev, ${ }^{\text {d }}$ Andrew N. Fitch $^{\mathrm{e}}$ and Vladimir V. \\ Chernyshev $^{\mathrm{a}, \mathrm{f}_{*}}$
}

\begin{abstract}
${ }^{a}$ A. N. Frumkin Institute of Physical Chemistry and Electrochemistry RAS, Leninsky prospect, 31, 119071 Moscow, Russian Federation, 'b Scientific Research Disinfectology Institute, Nauchnyi proezd 18, 117246 Moscow, Russian Federation, ${ }^{\mathbf{c} M o s c o w}$ Technological University, 78 Vernadsky Avenue, 119454 Moscow, Russian Federation, ${ }^{\mathbf{d}} \mathrm{V}$. N. Orekhovich Institute of Biomedical Chemistry, RAMS 10 build. 8, Pogodinskaya Street, 119121 Moscow, Russian Federation, ${ }^{\mathbf{E}}$ European Synchrotron Radiation Facility, BP 220, F-38043 Grenoble Cedex, France, and 'Department of Chemistry, M. V. Lomonosov Moscow State University, 119991 Moscow, Russian Federation. *Correspondence e-mail: vladimir@struct.chem.msu.ru
\end{abstract}

Porphyrin complexes of ruthenium are widely used as models for the heme protein system, for modelling naturally occurring iron-porphyrin systems and as catalysts in epoxidation reactions. The structural diversity of ruthenium complexes offers an opportunity to use them in the design of multifunctional supramolecular assemblies. Coproporphyrins and metallocoproporphyrins are used as sensors in bioassay and the potential use of derivatives as multiparametric sensors for oxygen and $\mathrm{H}^{+}$is one of the main factors driving a growing interest in the synthesis of new porphyrin derivatives. In the coproporphyrin I $\mathrm{Ru}^{\mathrm{II}}$ complex catena-poly[[carbonylruthenium(II)]- $\mu-2,7,12,17$ tetrakis[2-(ethoxycarbonyl)ethyl]-3,8,13,18-tetramethylporphyrinato- $\kappa^{5} N, N^{\prime}, N^{\prime \prime}$,$\left.N^{\prime \prime \prime}: O\right],\left[\mathrm{Ru}\left(\mathrm{C}_{44} \mathrm{H}_{52} \mathrm{~N}_{4} \mathrm{O}_{8}\right)(\mathrm{CO})\right]_{n}$, the $\mathrm{Ru}^{\text {II }}$ centre is coordinated by four $\mathrm{N}$ atoms in the basal plane, and by axial $\mathrm{C}$ (carbonyl ligand) and $\mathrm{O}$ (ethoxycarbonylethyl arm from a neighbouring complex) atoms. The complex adopts a distorted octahedral geometry. Self-assembly of the molecules during crystallization from a methylene chloride-ethanol $(1: 10 \mathrm{v} / \mathrm{v})$ solution at room temperature gives onedimensional polymeric chains.

\section{Introduction}

Porphyrin complexes of ruthenium have been studied actively in recent decades because of their many useful applications. They are widely used as models for the heme protein system (Masuda et al., 1982), for modelling naturally occurring ironporphyrin systems involved in oxidation processes of the mono- and dioxygenase type (James et al., 1988) and as catalysts in epoxidation reactions (Barona-Castano et al., 2016), including aerobic oxidation tandem epoxidation-isomerization (Jiang et al., 2008). Ruthenium in porphyrinates can exist in a variety of oxidation states, viz. $\mathrm{Ru}^{\mathrm{II}}$ (Ariel et al., 1984), $\mathrm{Ru}^{\mathrm{III}}$ (James et al., 1984), $\mathrm{Ru}^{\mathrm{IV}}$ (Maeda et al., 2015) or $\mathrm{Ru}^{\mathrm{VI}}$ (Katsunori et al., 2012), and it is able to coordinate one or two small axial ligands, such as aqua, hydroxide, dinitrogen, nitrite, nitrosyl, carbonyl, methanol, ethanol, tetrahydrofuran, pyridine and many others, as can be found from a search in the Cambridge Structural Database (ConQuest, Version 1.18 with updates; Groom et al., 2016), which gave 225 hits. The structural diversity of ruthenium complexes, which can also form metal-metal bonds (Collman et al., 1984), offers an opportunity to use them in the design of multifunctional supramolecular assemblies (Mamardashvili et al., 2013). 
Due to their unique photophysical and photochemical properties, coproporphyrins and metallocoproporphyrins have also been used as sensors in bioassay (Papkovsky \& O'Riordan, 2005; Dmitriev et al., 2012; Burke et al., 2007). The potential use of derivatives of coproporphyrins as multiparametric sensors for oxygen and $\mathrm{H}^{+}$is one of the main factors driving a growing interest in the synthesis of new porphyrin derivatives (Borchert et al., 2011). Recently, we reported synthetic and crystallographic studies of the coproporphyrin I molecular complexes with $\mathrm{Pd}^{\mathrm{II}}$ (Volov et al., 2014; Tyurin et al., 2015). We report herein the synthesis and structural characterization of the novel ruthenium(II) complex of coproporphyrin I tetraethyl ester, (1), namely catena-poly[[carbonylruthenium(II)]- $\mu$-2,7,12,17-tetrakis[2(ethoxycarbonyl)ethyl]-3,8,13,18-tetramethylporphyrinato$\left.\kappa^{5} N, N^{\prime}, N^{\prime \prime}, N^{\prime \prime \prime}: O\right],(2)$, which crystallizes with the formation of one-dimensional polymeric chains.

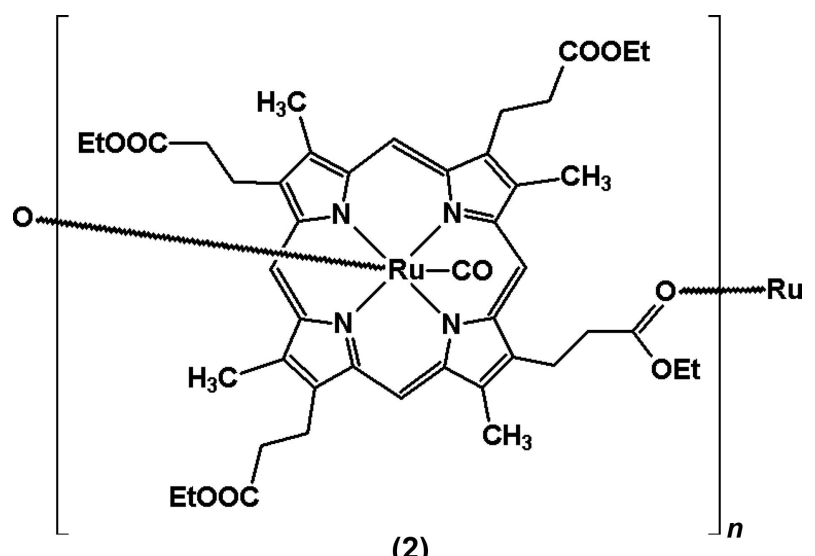

(2)

\section{Experimental}

Commercial reagents were used without purification. Solvents were purified according to standard procedures: $\mathrm{CH}_{2} \mathrm{Cl}_{2}$ was distilled over calcium hydride under an argon atmosphere and toluene was dried over magnesium chloride and then distilled over sodium. Coproporphyrin I, (1), was prepared according to a literature procedure (Smith, 1972). Silica gel 40/60 (Merck) was used for column and flash chromatography. For preparative thin-layer chromatography (TLC), silica gel 60 (Merck) $20 \times 20 \mathrm{~cm}$ plates with a $1 \mathrm{~mm}$ layer thickness were used. A mixture of methylene chloride-ethanol was used as solvent for elution. Electronic absorption, NMR and mass spectra for the final product are presented in the Supporting information.

\subsection{Synthesis and crystallization}

To a solution of (1) $(50 \mathrm{mg}, 0.065 \mathrm{mmol})$ in dry toluene $(15 \mathrm{ml})$ was added $\mathrm{Ru}_{3}(\mathrm{CO})_{12}(83.5 \mathrm{mg}, 0.13 \mathrm{mmol})$. The resulting mixture was refluxed for $24 \mathrm{~h}$. After completion of the reaction, the solvent was evaporated. Purification was carried out by preparative chromatography using an ethanoldichloromethane (100:1 v/v) mixture. A powder sample suitable for crystal structure determination was obtained by
Table 1

Experimental details.

\begin{tabular}{ll}
\hline Crystal data & \\
Chemical formula & {$\left[\mathrm{Ru}\left(\mathrm{C}_{44} \mathrm{H}_{52} \mathrm{~N}_{4} \mathrm{O}_{8}\right)(\mathrm{CO})\right]$} \\
$M_{\mathrm{r}}$ & 893.98 \\
Crystal system, space group & Monoclinic, $C 2 / c$ \\
Temperature $(\mathrm{K})$ & 295 \\
$a, b, c(\AA)$ & $39.3876(19), 9.6153(8)$, \\
& $29.5299(16)$ \\
$\beta\left({ }^{\circ}\right)$ & $130.719(7)$ \\
$V\left(\AA^{3}\right)$ & $8476.3(9)$ \\
$Z$ & 8 \\
Radiation type $_{\mu\left(\mathrm{mm}^{-1}\right)}$ & Synchrotron, $\lambda=0.399927(2) \AA$ \\
Specimen shape, size $(\mathrm{mm})$ & 0.09 \\
& Cylinder, $15 \times 1.0$
\end{tabular}

Data collection

Diffractometer

Specimen mounting

ESRF powder diffractometer ID22

Specimen was sealed in a $1.0 \mathrm{~mm}$ diameter borosilicate glass capillary

Data collection mode

Scan method

$2 \theta$ values $\left({ }^{\circ}\right)$

Transmission

Continuous

$2 \theta_{\text {min }}=1.000,2 \theta_{\max }=20.000$, $2 \theta_{\text {step }}=0.002$

Refinement

$R$ factors and goodness of fit

$R_{\mathrm{p}}=0.031, R_{\mathrm{wp}}=0.040, R_{\exp }=$

247

No. of parameters

No. of restraints

$\mathrm{H}$-atom treatment

193

$\mathrm{H}$-atom parameters not refined

Computer programs: MRIA (Zlokazov \& Chernyshev, 1992), FOX (Favre-Nicolin \& Cerný, 2004), PLATON (Spek, 2009), Mercury (Macrae et al., 2008), SHELXL97 (Sheldrick, 2008) and publCIF (Westrip, 2010).

crystallization from the methylene chloride-ethanol $(1: 10 \mathrm{v} / \mathrm{v})$ solvent system at room temperature over a period of $7 \mathrm{~d}$.

\subsection{Refinement}

X-ray powder diffraction measurements were carried out at room temperature at beamline ID22 of the European Synchrotron Radiation Facility (ESRF, Grenoble, France). The instrument is equipped with a cryogenically cooled double-crystal Si 111 monochromator and Si 111 analyzers. The powder was loaded into a $1 \mathrm{~mm}$ diameter borosilicate thin-walled glass capillary which was rotated during measurements at a rate of $1200 \mathrm{rpm}$ to improve the powder averaging. Calibration of the instrument and refinement of the X-ray wavelength $[0.399927$ (3) Å] were performed using NIST silicon standard 640c.

The monoclinic cell dimensions were determined using three indexing programs: TREOR90 (Werner et al., 1985), ITO (Visser, 1969) and AUTOX (Zlokazov, 1992, 1995). Based on systematic extinctions and other considerations, the space group was determined as $C 2 / c$. The unit-cell parameters and space group were further tested using a Pawley fit (Pawley, 1981) and confirmed by crystal structure solution. The geometry of the initial molecular model of (2) with the $\mathrm{Ru}$ centre coordinated by the four $\mathrm{N}$ atoms only, i.e. without the carbonyl ligand, was optimized by density functional theory (DFT) calculations in vacuo using the quantum-chemical program PRIRODA (Laikov, 1997, 2004, 2005; Laikov \& 


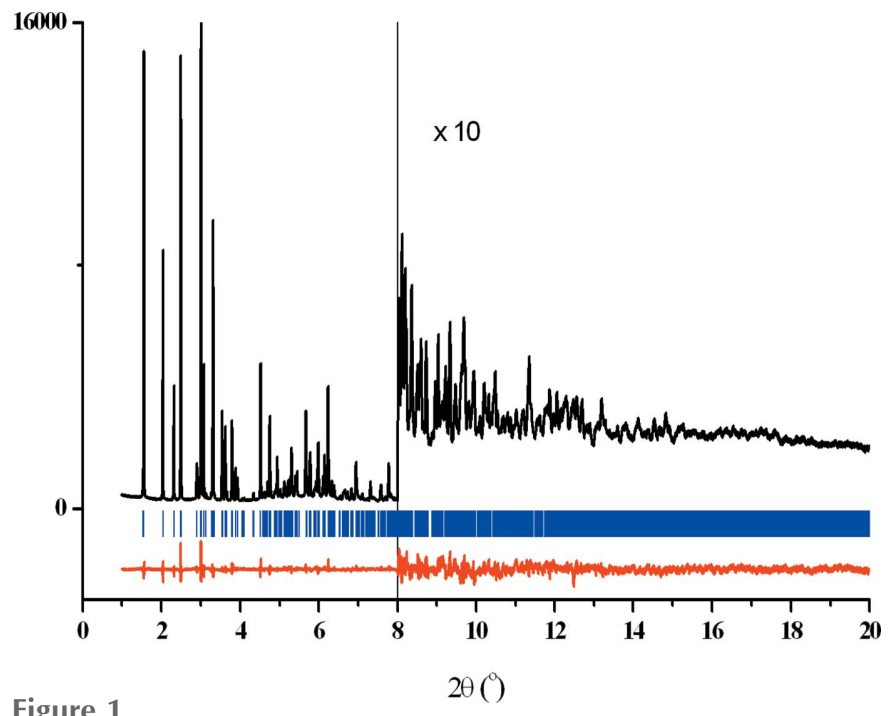

Figure 1

Rietveld plot showing the experimental (black) and difference (red) profiles for (2). The vertical bars (blue) correspond to the positions of the Bragg peaks.

Ustynyuk, 2005), employing the generalized gradient approximation (GGA) and the PBE exchange-correlation function (Perdew et al., 1996). This fragment was used in the crystal structure determination. The crystal structure was solved using two techniques, viz. simulated annealing (Zhukov et al., 2001) using the program MRIA (Zlokazov \& Chernyshev, 1992) and parallel tempering (Favre-Nicolin \& Cerný, 2002) using the program FOX (Favre-Nicolin \& Cerný, 2002, 2004). The carbonyl group was added to the model after the analysis of a difference Fourier map, where axial positive residual electron density was observed at $1.9 \AA$ from the $\mathrm{Ru}$ centre. The final Rietveld refinement was performed with the program MRIA following the procedure described by us previously (Dorokhov et al., 2007; Logacheva et al., 2009; Chernyshev et al., 2013). In the refinement, anisotropic line broadening was taken into account with the use of nine variables (Popa, 1998). All non-H atoms were refined isotropically. One common $U_{\text {iso }}$ parameter was varied for 32 atoms, i.e. 24 atoms of the porphyrin core and eight $\mathrm{C}$ atoms attached to the core. The maximum difference peak and hole at the end of

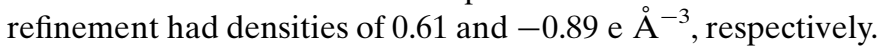
Crystal data, data collection and structure refinement details are summarized in Table 1 and the diffraction profiles after the final bond-restrained Rietveld refinement are shown in Fig. 1.

\section{Results and discussion}

In the title compound, the $\mathrm{Ru}^{\mathrm{II}}$ centre is coordinated by four $\mathrm{N}$ atoms $[\mathrm{Ru}-\mathrm{N}=2.025(17)-2.055(12) \AA]$ in the basal plane and by an axial carbonyl ligand $[\mathrm{Ru}-\mathrm{C}=1.871$ (19) $\AA$ ก $]$. A carbonyl $\mathrm{O}$ atom (O5) of the ethoxycarbonylethyl arm $[\mathrm{Ru}-\mathrm{O}=2.194$ (12) $\mathrm{A}]$ from a neighbouring asymmetric unit completes the distorted octahedral coordination geometry. The metal atom is displaced by 0.11 (1) $\AA$ from the mean plane of the porphyrin core, toward the carbonyl ligand. As seen in Fig. 2, just one of four ethyl ester arms serves as a connector between neighbouring molecules via the aforementioned axially coordinating atom O5, mediating the formation of polymeric chains running along [010] (Fig. 3). The porphyrin cores of neighbouring links in the chain are inclined to one another by $74.3(2)^{\circ}$. Examples of metalporphyrin complexes in which the carbonyl groups of lateral substituents coordinate neighbouring metal centres have been observed, though they are rare; our search in the Cambridge Structural Database (CSD) resulted in just three hits, namely catena-(bis $\left\{\mu_{3}-5,10,15,20\right.$-tetrakis [3', $3^{\prime}$-bis(ethoxycarbonyl)phenyl]porphyrinato\}dizinc(II)) (CSD refcode LUBKOI; Bhyrappa et al., 2002), bis $\left\{\mu_{2}\right.$-diethyl-2,2'-[(porphyrin-5,15diyl)bis(naphthalene-1,2-diyloxy)]diacetatato\}dizinc(II) dichloromethane solvate (MUVQAW; Amaya et al., 2010) and catena-[bis $\left\{\mu\right.$-5-tert-butyl- $N, N^{\prime}$-bis[2-(10,15,20-triphenylporphyrin-21,23-diyl-5-yl)phenyl]isophthalamide\}tetrazinc unknown solvate] (OGUFIH; Fang et al., 2015).

The most interesting feature of the crystal structure of (2) is the formation of a molecular cavity, as the four ethoxycarbonylethyl substituents are located on the same side of the porphyrin plane, like the petals of a tulip. The CSD contains no examples of tetramethyl metalloporphyrins with four ethoxycarbonylethyl branches, and a search for metalloporphyrins with similar substituents yielded the following four structures: $\{2,7,12,17$-tetrakis[2-(isopropyloxycarbonyl)ethyl]3,8,13,18-tetramethylporphyrinato\}platinum(II) and -palladium(II) (KILQIH and KILQON, respectively; Zamilatskov et al., 2012), and chlorido- and (acetato- $\kappa^{2} O, O^{\prime}$ )(tetramethyl3,8,13,18-tetramethylporphyrin-2,7,12,17-tetrapropionato- $\kappa^{4} N$,$N^{\prime}, N^{\prime \prime}, N^{\prime \prime \prime}$ )thallium(III) dichloromethane solvate (WECSIG and WECTAZ, respectively; Senge et al., 1993); none of these structures has a molecular cavity. Surprisingly, to provide the axial coordination of the $\mathrm{Ru}^{\mathrm{II}}$ atom in (2), the flexible ethoxycarbonylethyl branch from the neighbouring segment of the polymer positions its $\mathrm{O}$ atom (O5) within the 'tulip petals'. This

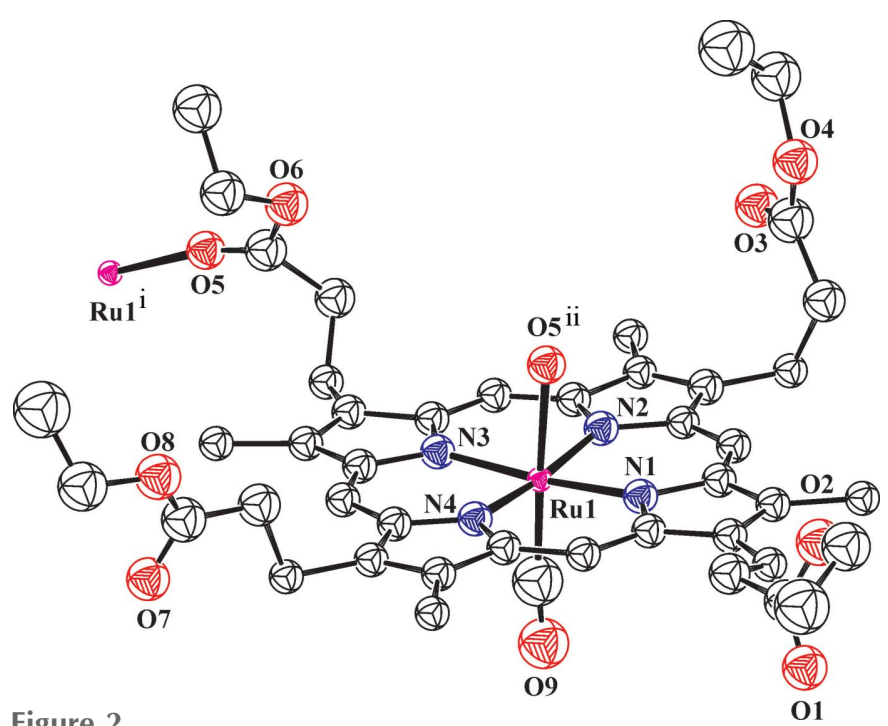

View of (2) showing the atomic numbering and 50\% probability displacement spheres. $\mathrm{H}$ atoms have been omitted for clarity. [Symmetry codes: (i) $-x+\frac{3}{2}, y+\frac{1}{2},-z+\frac{3}{2}$; (ii) $-x+\frac{3}{2}, y-\frac{1}{2},-z+\frac{3}{2}$.] 


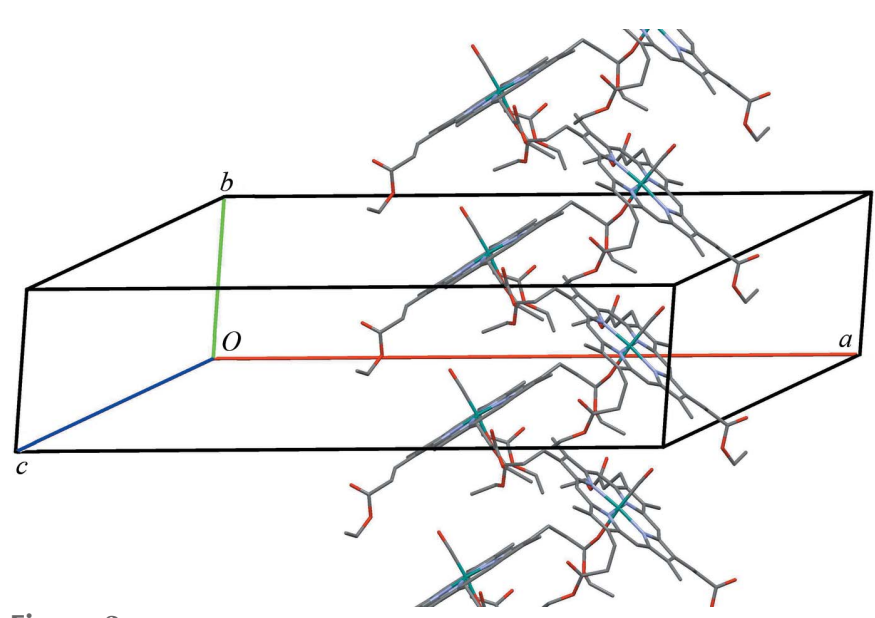

Figure 3

A portion of the polymeric chain in the crystal structure of (2).

fragment of the neighbouring porphyrin core thus penetrates into the 'tulip bowl' of the molecule (Fig. 3).

The aforementioned features of the crystal packing of (2) demonstrate its potential for self-assembling in various modes depending on crystallization and environmental conditions, as has been observed for [tetrakis(pyridin-4-yl)porphyrin]zinc, [Zn(tpyp)]. Several coordination polymers were obtained for [Zn(tpyp)], viz. a one-dimensional chain structure (Krupitsky et al., 1994), a one-dimensional ladder structure (DiskinPosner et al., 2001), a one-dimensional ribbon structure (Ring et al., 2005) and a three-dimensional framework (Krupitsky et al., 1994). For compound (2), we hope to remove the carbonyl fragments either from the solution before crystallization or directly from the solid-state sample to explore other possibilities for self-assembly of the coproporphyrin I tetraethyl ester ruthenium(II) complex.

\section{Acknowledgements}

This research was supported by the Russian Ministry of Science and Education (grant No. RFMEFI61616X0069). We also thank ESRF for the access to ID22 station, experiment MA-3313.

\section{References}

Amaya, T., Ueda, T. \& Hirao, T. (2010). Tetrahedron Lett. 51, 33763379.

Ariel, S., Dolphin, D., Donazetis, G., James, B. R., Leung, T. W., Rettig, S. J., Trotter, J. \& Williams, G. M. (1984). Can. J. Chem. 62, 755-762.

Barona-Castano, J. C., Carmona-Vargas, C. C., Brocksom, T. J. \& de Oliveira, K. T. (2016). Molecules, 21, 310-337.

Bhyrappa, P., Vaijayanthimala, G. \& Verghese, B. (2002). Tetrahedron Lett. 43, 6427-6429.

Borchert, N. B., Ponomarev, G. V., Kerry, J. P. \& Papkovsky, D. B. (2011). Anal. Chem. 83, 18-22.

Burke, M., O'Sullivan, P. J., Ponomarev, G. V., Yashunsky, D. V. \& Papkovsky, D. B. (2007). Anal. Chim. Acta, 585, 139-146.

Chernyshev, V. V., Petkune, S., Actins, A., Auzins, R., Davlyatshin, D. I., Nosyrev, P. V. \& Velikodny, Y. A. (2013). Acta Cryst. C69, 299302.
Collman, J. P., Barnes, C. E., Swepston, P. N. \& Ibers, J. A. (1984). J. Am. Chem. Soc. 106, 3500-3510.

Diskin-Posner, Y., Patra, G. K. \& Goldberg, I. (2001). J. Chem. Soc. Dalton Trans. pp. 2775-2782.

Dmitriev, R. I., Zhdanov, A. V., Jasionek, G. \& Papkovsky, D. B. (2012). Anal. Chem. 84, 2930-2938.

Dorokhov, A. V., Chernyshov, D. Y., Burlov, A. S., Garnovskii, A. D., Ivanova, I. S., Pyatova, E. N., Tsivadze, A. Y., Aslanov, L. A. \& Chernyshev, V. V. (2007). Acta Cryst. B63, 402-410.

Fang, X. S., Han, Z., Xu, C. L., Li, X. H., Wang, Y. \& Hu, C. J. (2015). Dalton Trans. 44, 12511-12515.

Favre-Nicolin, V. \& Cerný, R. (2002). J. Appl. Cryst. 35, 734-743.

Favre-Nicolin, V. \& Cerný, R. (2004). Z. Kristallogr. 219, 847-856.

Groom, C. R., Bruno, I. J., Lightfoot, M. P. \& Ward, S. C. (2016). Acta Cryst. B72, 171-179.

James, B. R., Dolphin, D., Leung, T. W., Einstein, F. W. B. \& Willis, A. C. (1984). Can. J. Chem. 62, 1238-1245.

James, B. R., Pacheco, A., Rettig, S. J. \& Ibers, R. G. (1988). Inorg. Chem. 27, 2414-2421.

Jiang, G., Chen, J., Thu, H.-Y., Huang, J.-S., Zhu, N. \& Che, C.-M. (2008). Angew. Chem. Int. Ed. 47, 6638-6642.

Katsunori, T., Mazumder, K., Siwu, E. R. O., Nozaki, S., Watanabe, Ya. \& Fukase, K. (2012). Tetrahedron Lett. 53, 1756-1759.

Krupitsky, H., Stein, Z., Goldberg, I. \& Strouse, C. E. (1994). J. Inclusion Phenom. Mol. Recogn. Chem. 18, 177-192.

Laikov, D. N. (1997). Chem. Phys. Lett. 281, 151-154.

Laikov, D. N. (2004). PRIRODA. Moscow State University.

Laikov, D. N. (2005). Chem. Phys. Lett. 416, 116-120.

Laikov, D. N. \& Ustynyuk, Y. A. (2005). Russ. Chem. Bull. 54, 820826.

Logacheva, N. M., Baulin, V. E., Tsivadze, A. Y., Pyatova, E. N., Ivanova, I. S., Velikodny, Y. A. \& Chernyshev, V. V. (2009). Dalton Trans. pp. 2482-2489.

Macrae, C. F., Bruno, I. J., Chisholm, J. A., Edgington, P. R., McCabe, P., Pidcock, E., Rodriguez-Monge, L., Taylor, R., van de Streek, J. \& Wood, P. A. (2008). J. Appl. Cryst. 41, 466-470.

Maeda, K., Terada, T., Iwamoto, T., Kurahashi, T. \& Matsubara, S. (2015). Org. Lett. 17, 5284-5287.

Mamardashvili, G. M., Mamardashvili, N. Zh. \& Koifman, O. I. (2013). Macroheterocycles, 6, 67-73.

Masuda, H., Taga, T., Osaki, K., Sugimoto, H., Mori, M. \& Ogoshi, H. (1982). Bull. Chem. Soc. Jpn, 55, 3887-3890.

Papkovsky, D. B. \& O'Riordan, T. C. (2005). J. Fluoresc. 15, 569584.

Pawley, G. S. (1981). J. Appl. Cryst. 14, 357-361.

Perdew, J. P. S., Burke, S. \& Ernzerhof, M. (1996). Phys. Rev. Lett. 77, 3865-3868.

Popa, N. C. (1998). J. Appl. Cryst. 31, 176-180.

Ring, D. J., Aragoni, M. C., Champness, N. R. \& Wilson, C. (2005). CrystEngComm, 7, 621-623.

Senge, M. O., Ruhlandt-Senge, K., Regli, K. J. \& Smith, K. M. (1993). J. Chem. Soc. Dalton Trans. pp. 3519-3538.

Sheldrick, G. M. (2008). Acta Cryst. A64, 112-122.

Smith, K. M. (1972). J. Chem. Soc. Perkin Trans. 1, pp. 1471-1475.

Spek, A. L. (2009). Acta Cryst. D65, 148-155.

Tyurin, V. S., Erzina, D. R., Zamilatskov, I. A., Chernyadyev, A. Yu., Ponomarev, G. V., Yashunskiy, D. V., Maksimova, A. V., Krasnovskiy, A. A. \& Tsivadze, A. Yu. (2015). Macroheterocycles, 8, 376-383.

Visser, J. W. (1969). J. Appl. Cryst. 2, 89-95.

Volov, A. N., Zamilatskov, I. A., Mikhel, I. S., Erzina, D. R., Ponomarev, G. V., Koifman, O. I. \& Tsivadze, A. Yu. (2014). Macroheterocycles, 7, 256-261.

Werner, P.-E., Eriksson, L. \& Westdahl, M. (1985). J. Appl. Cryst. 18, 367-370.

Westrip, S. P. (2010). J. Appl. Cryst. 43, 920-925.

Zamilatskov, I. A., Savinkina, E. V., Volov, A. N., Grigoriev, M. S., Lonin, I. S., Obolenskaya, L. N., Ponomarev, G. V., Koifman, O. I., 
Kuzovlev, A. S., Kuzmicheva, G. M. \& Tsivadze, A. Yu. (2012). Macroheterocycles, 5, 308-314.

Zhukov, S. G., Chernyshev, V. V., Babaev, E. V., Sonneveld, E. J. \& Schenk, H. (2001). Z. Kristallogr. 216, 5-9.
Zlokazov, V. B. (1992). J. Appl. Cryst. 25, 69-72.

Zlokazov, V. B. (1995). Comput. Phys. Commun. 85, 415-422.

Zlokazov, V. B. \& Chernyshev, V. V. (1992). J. Appl. Cryst. 25, 447451. 


\section{supporting information}

Acta Cryst. (2017). C73, 47-51 [https://doi.org/10.1107/S2053229616019422]

\section{Polymeric structure of a coproporphyrin I ruthenium(II) complex: a powder diffraction study}

\section{Sergey V. Andreev, Sergey A. Zverev, Ilya A. Zamilatskov, Nadezhda M. Kurochkina, Gelii V. Ponomarev, Andrew N. Fitch and Vladimir V. Chernyshev}

\section{Computing details}

Cell refinement: MRIA (Zlokazov \& Chernyshev, 1992); program(s) used to solve structure: MRIA (Zlokazov \& Chernyshev, 1992) and FOX (Favre-Nicolin \& Cerný, 2004); program(s) used to refine structure: MRIA (Zlokazov \& Chernyshev, 1992); molecular graphics: PLATON (Spek, 2009) and Mercury (Macrae et al., 2008); software used to prepare material for publication: SHELXL97 (Sheldrick, 2008) and publCIF (Westrip, 2010).

catena-Poly[[carbonylruthenium(II)]- $\mu$-2,7,12,17-tetrakis[2-(ethoxycarbonyl)ethyl]-3,8,13,18tetramethylporphyrinato- $\left.\kappa^{5} N, N^{\prime}, N^{\prime \prime}, N^{\prime \prime \prime}: O\right]$

Crystal data

$\left[\mathrm{Ru}\left(\mathrm{C}_{44} \mathrm{H}_{52} \mathrm{~N}_{4} \mathrm{O}_{8}\right)(\mathrm{CO})\right]$

$M_{r}=893.98$

Monoclinic, $C 2 / c$

Hall symbol: $-\mathrm{C} 2 \mathrm{yc}$

$a=39.3876(19) \AA$

$b=9.6153(8) \AA$

$c=29.5299(16) \AA$

$\beta=130.719(7)^{\circ}$

$V=8476.3(9) \AA^{3}$

$Z=8$

Data collection

ESRF powder diffractometer ID22

Radiation source: ID22 bending magnet at ESRF, synchrotron radiation

Si 111 double crystal monochromator

\section{Refinement}

Refinement on $I_{\text {net }}$

Least-squares matrix: full with fixed elements per cycle

$R_{\mathrm{p}}=0.031$

$R_{\text {wp }}=0.040$

$R_{\text {exp }}=0.013$

$R_{\text {Bragg }}=0.070$

9501 data points

Profile function: split-type pseudo-Voigt
$F(000)=3728$

$D_{\mathrm{x}}=1.401 \mathrm{Mg} \mathrm{m}^{-3}$

Synchrotron radiation, $\lambda=0.399927(2) \AA$

$\mu=0.09 \mathrm{~mm}^{-1}$

$T=295 \mathrm{~K}$

Particle morphology: no specific habit colorless

cylinder, $15 \times 1.0 \mathrm{~mm}$

Specimen preparation: Prepared at $295 \mathrm{~K}$ and $101 \mathrm{kPa}$

Specimen mounting: Specimen was sealed in a $1.0 \mathrm{~mm}$ diameter borosilicate glass capillary Data collection mode: transmission

Scan method: continuous

$2 \theta_{\text {min }}=1.000^{\circ}, 2 \theta_{\max }=20.000^{\circ}, 2 \theta_{\text {step }}=0.002^{\circ}$

247 parameters

193 restraints

21 constraints

$\mathrm{H}$-atom parameters not refined

Weighting scheme based on measured s.u.'s

$(\Delta / \sigma)_{\max }=0.003$

Background function: Chebyshev polynomial up to the 5 th order

Preferred orientation correction: none 


\section{Special details}

Geometry. All esds (except the esd in the dihedral angle between two 1.s. planes) are estimated using the full covariance matrix. The cell esds are taken into account individually in the estimation of esds in distances, angles and torsion angles; correlations between esds in cell parameters are only used when they are defined by crystal symmetry. An approximate (isotropic) treatment of cell esds is used for estimating esds involving l.s. planes.

Fractional atomic coordinates and isotropic or equivalent isotropic displacement parameters $\left(\AA^{2}\right)$

\begin{tabular}{|c|c|c|c|c|}
\hline & $x$ & $y$ & $z$ & $U_{\text {iso }} * / U_{\text {eq }}$ \\
\hline Ru1 & $0.88520(4)$ & $0.5249(2)$ & $0.82080(6)$ & $0.0170(12)^{*}$ \\
\hline N1 & $0.9371(3)$ & $0.3887(14)$ & $0.8584(5)$ & $0.034(10)^{*}$ \\
\hline N2 & 0.8917 (3) & $0.5230(13)$ & $0.8947(5)$ & $0.034(10)^{*}$ \\
\hline N3 & $0.8290(3)$ & $0.6456(15)$ & $0.7803(5)$ & $0.034(10)^{*}$ \\
\hline N4 & $0.8732(3)$ & $0.5119(13)$ & $0.7428(5)$ & $0.034(10)^{*}$ \\
\hline $\mathrm{C} 1$ & 0.9549 (4) & $0.3341(19)$ & $0.8342(6)$ & $0.034(10)^{*}$ \\
\hline $\mathrm{C} 2$ & 0.9928 (4) & $0.2503(19)$ & $0.8768(6)$ & $0.034(10)^{*}$ \\
\hline $\mathrm{C} 3$ & 0.9990 (4) & $0.2549(18)$ & $0.9290(7)$ & $0.034(10)^{*}$ \\
\hline $\mathrm{C} 4$ & $0.9652(4)$ & $0.3374(19)$ & $0.9178(6)$ & $0.034(10)^{*}$ \\
\hline $\mathrm{C} 5$ & 0.9575 (4) & $0.3725(18)$ & $0.9559(5)$ & $0.034(10)^{*}$ \\
\hline H5 & 0.9776 & 0.3334 & 0.9938 & $0.041 *$ \\
\hline $\mathrm{C} 6$ & 0.9254 (4) & $0.4549(18)$ & $0.9473(6)$ & $0.034(10)^{*}$ \\
\hline $\mathrm{C} 7$ & 0.9209 (4) & $0.4849(18)$ & $0.9914(6)$ & $0.034(10)^{*}$ \\
\hline $\mathrm{C} 8$ & 0.8857 (4) & $0.5682(17)$ & $0.9663(6)$ & $0.034(10)^{*}$ \\
\hline C9 & 0.8675 (4) & 0.5963 (19) & $0.9058(6)$ & $0.034(10)^{*}$ \\
\hline $\mathrm{C} 10$ & 0.8307 (4) & 0.6775 (19) & $0.8638(6)$ & $0.034(10)^{*}$ \\
\hline H10 & 0.8167 & 0.7226 & 0.8756 & $0.041^{*}$ \\
\hline C11 & 0.8129 (4) & $0.6974(17)$ & $0.8053(6)$ & $0.034(10)^{*}$ \\
\hline $\mathrm{C} 12$ & 0.7734 (4) & $0.7848(19)$ & $0.7630(6)$ & $0.034(10)^{*}$ \\
\hline $\mathrm{C} 13$ & $0.7672(4)$ & $0.7845(18)$ & $0.7121(6)$ & $0.034(10)^{*}$ \\
\hline $\mathrm{C} 14$ & 0.8005 (4) & $0.6945(18)$ & $0.7212(6)$ & $0.034(10)^{*}$ \\
\hline C15 & $0.8070(4)$ & $0.6615(19)$ & $0.6821(5)$ & $0.034(10)^{*}$ \\
\hline H15 & 0.7866 & 0.7008 & 0.6443 & $0.041 *$ \\
\hline $\mathrm{C} 16$ & 0.8397 (4) & $0.5778(19)$ & $0.6906(6)$ & $0.034(10)^{*}$ \\
\hline $\mathrm{C} 17$ & 0.8442 (4) & $0.5503(17)$ & $0.6468(6)$ & $0.034(10)^{*}$ \\
\hline $\mathrm{C} 18$ & 0.8800 & $0.4628(17)$ & $0.6723(6)$ & $0.034(10)^{*}$ \\
\hline C19 & 0.8980 & 0.4395 (19) & $0.7331(6)$ & $0.034(10)^{*}$ \\
\hline $\mathrm{C} 20$ & $0.9356(4)$ & $0.3604(19)$ & $0.7753(6)$ & $0.034(10)^{*}$ \\
\hline $\mathrm{H} 20$ & 0.9497 & 0.3198 & 0.7628 & $0.041 *$ \\
\hline $\mathrm{C} 21$ & $1.0222(5)$ & $0.1708(18)$ & $0.8744(7)$ & $0.034(10)^{*}$ \\
\hline $\mathrm{H} 21 \mathrm{~A}$ & 1.0297 & 0.0874 & 0.8978 & $0.041 *$ \\
\hline $\mathrm{H} 21 \mathrm{~B}$ & 1.0497 & 0.2240 & 0.8954 & $0.041^{*}$ \\
\hline $\mathrm{C} 22$ & 1.0099 (4) & $0.126(2)$ & $0.8183(6)$ & $0.049(10)^{*}$ \\
\hline $\mathrm{H} 22 \mathrm{~A}$ & 1.0048 & 0.2078 & 0.7952 & $0.059 *$ \\
\hline $\mathrm{H} 22 \mathrm{~B}$ & 0.9821 & 0.0744 & 0.7956 & $0.059 *$ \\
\hline $\mathrm{C} 23$ & $1.0462(4)$ & $0.0347(18)$ & $0.8273(7)$ & $0.055(11)^{*}$ \\
\hline $\mathrm{C} 24$ & $1.0564(5)$ & $-0.214(2)$ & $0.8123(6)$ & $0.069(10)^{*}$ \\
\hline $\mathrm{H} 24 \mathrm{~A}$ & 1.0360 & -0.2920 & 0.7966 & $0.083^{*}$ \\
\hline H24B & 1.0856 & -0.2452 & 0.8478 & $0.083 *$ \\
\hline
\end{tabular}




\begin{tabular}{|c|c|c|c|c|}
\hline $\mathrm{C} 25$ & $1.0596(5)$ & $-0.168(2)$ & $0.7667(7)$ & $0.089(11)^{*}$ \\
\hline $\mathrm{H} 25 \mathrm{~A}$ & 1.0699 & -0.2447 & 0.7575 & $0.134^{*}$ \\
\hline $\mathrm{H} 25 \mathrm{~B}$ & 1.0803 & -0.0925 & 0.7823 & $0.134^{*}$ \\
\hline $\mathrm{H} 25 \mathrm{C}$ & 1.0306 & -0.1392 & 0.7311 & $0.134 *$ \\
\hline $\mathrm{C} 26$ & $1.0361(4)$ & $0.1737(18)$ & $0.9841(6)$ & $0.034(10)^{*}$ \\
\hline $\mathrm{H} 26 \mathrm{~A}$ & 1.0538 & 0.1275 & 0.9770 & $0.051^{*}$ \\
\hline H26B & 1.0233 & 0.1058 & 0.9930 & $0.051 *$ \\
\hline $\mathrm{H} 26 \mathrm{C}$ & 1.0547 & 0.2362 & 1.0173 & $0.051 *$ \\
\hline $\mathrm{C} 27$ & $0.9516(4)$ & $0.4256(18)$ & $1.0535(6)$ & $0.034(10)^{*}$ \\
\hline $\mathrm{H} 27 \mathrm{~A}$ & 0.9472 & 0.4749 & 1.0780 & $0.041^{*}$ \\
\hline H27B & 0.9825 & 0.4390 & 1.0710 & $0.041^{*}$ \\
\hline C28 & 0.9429 (4) & $0.2704(18)$ & $1.0533(6)$ & $0.051(10)^{*}$ \\
\hline $\mathrm{H} 28 \mathrm{~A}$ & 0.9447 & 0.2243 & 1.0257 & $0.061^{*}$ \\
\hline $\mathrm{H} 28 \mathrm{~B}$ & 0.9669 & 0.2337 & 1.0928 & $0.061^{*}$ \\
\hline C29 & 0.8992 (4) & $0.2311(19)$ & $1.0369(6)$ & $0.059(11)^{*}$ \\
\hline $\mathrm{C} 30$ & $0.8427(5)$ & $0.0444(18)$ & $1.0068(7)$ & $0.071(11)^{*}$ \\
\hline $\mathrm{H} 30 \mathrm{~A}$ & 0.8262 & 0.1176 & 1.0078 & $0.085^{*}$ \\
\hline $\mathrm{H} 30 \mathrm{~B}$ & 0.8528 & -0.0222 & 1.0381 & $0.085^{*}$ \\
\hline C31 & 0.8128 & $-0.0275(16)$ & $0.9454(5)$ & $0.091(12)^{*}$ \\
\hline $\mathrm{H} 31 \mathrm{~A}$ & 0.7895 & 0.0350 & 0.9158 & $0.136^{*}$ \\
\hline H31B & 0.7997 & -0.1095 & 0.9470 & $0.136^{*}$ \\
\hline $\mathrm{H} 31 \mathrm{C}$ & 0.8306 & -0.0529 & 0.9350 & $0.136^{*}$ \\
\hline C32 & 0.8667 (4) & $0.6283(19)$ & $0.9920(6)$ & $0.034(10)^{*}$ \\
\hline $\mathrm{H} 32 \mathrm{~A}$ & 0.8411 & 0.6845 & 0.9624 & $0.051^{*}$ \\
\hline H32B & 0.8889 & 0.6848 & 1.0260 & $0.051^{*}$ \\
\hline $\mathrm{H} 32 \mathrm{C}$ & 0.8578 & 0.5545 & 1.0042 & $0.051 *$ \\
\hline C33 & 0.7508 (4) & $0.859(2)$ & $0.7794(6)$ & $0.034(10)^{*}$ \\
\hline $\mathrm{H} 33 \mathrm{~A}$ & 0.7311 & 0.9267 & 0.7482 & $0.041^{*}$ \\
\hline H33B & 0.7734 & 0.9112 & 0.8155 & $0.041^{*}$ \\
\hline C34 & $0.7244(5)$ & $0.7836(17)$ & $0.7898(7)$ & $0.046(10) *$ \\
\hline $\mathrm{H} 34 \mathrm{~A}$ & 0.7200 & 0.8411 & 0.8126 & $0.055^{*}$ \\
\hline H34B & 0.7402 & 0.7001 & 0.8127 & $0.055^{*}$ \\
\hline C35 & $0.6781(4)$ & $0.7437(17)$ & $0.7289(6)$ & $0.050(10)^{*}$ \\
\hline C36 & $0.6421(4)$ & $0.5757(18)$ & $0.6487(7)$ & $0.058(10)^{*}$ \\
\hline $\mathrm{H} 36 \mathrm{~A}$ & 0.6347 & 0.6607 & 0.6262 & $0.070^{*}$ \\
\hline H36B & 0.6569 & 0.5129 & 0.6406 & $0.070 *$ \\
\hline C37 & 0.6008 (4) & $0.5101(18)$ & $0.6313(7)$ & $0.068(12)^{*}$ \\
\hline H37A & 0.5807 & 0.4886 & 0.5894 & $0.102 *$ \\
\hline H37B & 0.5865 & 0.5733 & 0.6395 & $0.102^{*}$ \\
\hline $\mathrm{H} 37 \mathrm{C}$ & 0.6086 & 0.4261 & 0.6538 & $0.102 *$ \\
\hline C38 & $0.7278(4)$ & $0.8624(18)$ & $0.6580(6)$ & $0.034(10)^{*}$ \\
\hline H38A & 0.7279 & 0.8526 & 0.6258 & $0.051^{*}$ \\
\hline H38B & 0.7300 & 0.9591 & 0.6677 & $0.051^{*}$ \\
\hline $\mathrm{H} 38 \mathrm{C}$ & 0.7004 & 0.8250 & 0.6462 & $0.051 *$ \\
\hline C39 & 0.8137 (4) & $0.6012(19)$ & $0.5812(6)$ & $0.034(10)^{*}$ \\
\hline H39A & 0.7997 & 0.6887 & 0.5771 & $0.041^{*}$ \\
\hline H39B & 0.8313 & 0.6155 & 0.5693 & $0.041^{*}$ \\
\hline C40 & 0.7777 (4) & $0.4897(18)$ & $0.5414(6)$ & $0.052(12)^{*}$ \\
\hline
\end{tabular}




$\begin{array}{lllll}\text { H40A } & 0.7921 & 0.3996 & 0.5515 & 0.062^{*} \\ \text { H40B } & 0.7576 & 0.4866 & 0.5498 & 0.062^{*} \\ \text { C41 } & 0.7509(5) & 0.5157(17) & 0.4762(7) & 0.058(11)^{*} \\ \text { C42 } & 0.6974(4) & 0.4449(19) & 0.3694(7) & 0.071(11)^{*} \\ \text { H42A } & 0.6946 & 0.5437 & 0.3609 & 0.085^{*} \\ \text { H42B } & 0.7114 & 0.4020 & 0.3555 & 0.085^{*} \\ \text { C43 } & 0.6524(4) & 0.384(2) & 0.3371(6) & 0.094(11)^{*} \\ \text { H43A } & 0.6345 & 0.3992 & 0.2950 & 0.141^{*} \\ \text { H43B } & 0.6383 & 0.4276 & 0.3504 & 0.141^{*} \\ \text { H43C } & 0.6552 & 0.2860 & 0.3449 & 0.141^{*} \\ \text { C44 } & 0.8932(4) & 0.4175(19) & 0.6362(6) & 0.034(10)^{*} \\ \text { H44A } & 0.9187 & 0.3568 & 0.6600 & 0.051^{*} \\ \text { H44B } & 0.9006 & 0.4979 & 0.6249 & 0.051^{*} \\ \text { H44C } & 0.8686 & 0.3692 & 0.6010 & 0.051^{*} \\ \text { O1 } & 1.0781(3) & 0.0868(13) & 0.8400(4) & 0.058(7)^{*} \\ \text { O2 } & 1.0411(3) & -0.1050(14) & 0.8286(4) & 0.063(7)^{*} \\ \text { O3 } & 0.8775(3) & 0.3143(12) & 1.0409(4) & 0.054(7)^{*} \\ \text { O4 } & 0.8818(3) & 0.1038(13) & 1.0157(4) & 0.058(7)^{*} \\ \text { O5 } & 0.6509(3) & 0.8273(12) & 0.7038(4) & 0.042(6)^{*} \\ \text { O6 } & 0.6724(3) & 0.6071(13) & 0.7148(4) & 0.053(6)^{*} \\ \text { O7 } & 0.7559(3) & 0.6281(14) & 0.4631(4) & 0.054(6)^{*} \\ \text { O8 } & 0.7260(3) & 0.4236(13) & 0.4346(4) & 0.060(7)^{*} \\ \text { O9 } & 0.9432(3) & 0.7812(12) & 0.8590(4) & 0.074(7)^{*} \\ \text { C45 } & 0.9192(5) & 0.6859(19) & 0.8412(6) & 0.074(10)^{*} \\ & & & & \end{array}$

Geometric parameters $\left(A,{ }^{\circ}\right)$

\begin{tabular}{llll}
\hline $\mathrm{Ru} 1-\mathrm{C} 45$ & $1.871(19)$ & $\mathrm{C} 25-\mathrm{H} 25 \mathrm{C}$ & 0.9600 \\
$\mathrm{Ru} 1-\mathrm{N} 2$ & $2.025(17)$ & $\mathrm{C} 26-\mathrm{H} 26 \mathrm{~A}$ & 0.9601 \\
$\mathrm{Ru} 1-\mathrm{N} 4$ & $2.031(16)$ & $\mathrm{C} 26-\mathrm{H} 26 \mathrm{~B}$ & 0.9599 \\
$\mathrm{Ru} 1-\mathrm{N} 1$ & $2.041(12)$ & $\mathrm{C} 26-\mathrm{H} 26 \mathrm{C}$ & 0.9600 \\
$\mathrm{Ru} 1-\mathrm{N} 3$ & $2.055(12)$ & $\mathrm{C} 27-\mathrm{C} 28$ & $1.53(2)$ \\
$\mathrm{Ru} 1-\mathrm{O} 5$ & $\mathrm{i}$ & $\mathrm{C} 27-\mathrm{H} 27 \mathrm{~A}$ & 0.9700 \\
$\mathrm{~N} 1-\mathrm{C} 1$ & $2.194(12)$ & $\mathrm{C} 27-\mathrm{H} 27 \mathrm{~B}$ & 0.9700 \\
$\mathrm{~N} 1-\mathrm{C} 4$ & $1.39(3)$ & $\mathrm{C} 28-\mathrm{C} 29$ & $1.50(3)$ \\
$\mathrm{N} 2-\mathrm{C} 6$ & $1.418(18)$ & $\mathrm{C} 28-\mathrm{H} 28 \mathrm{~A}$ & 0.9700 \\
$\mathrm{~N} 2-\mathrm{C} 9$ & $1.383(17)$ & $\mathrm{C} 28-\mathrm{H} 28 \mathrm{~B}$ & 0.9700 \\
$\mathrm{~N} 3-\mathrm{C} 11$ & $1.39(3)$ & $\mathrm{C} 29-\mathrm{O} 3$ & $1.23(2)$ \\
$\mathrm{N} 3-\mathrm{C} 14$ & $1.34(3)$ & $\mathrm{C} 29-\mathrm{O} 4$ & $1.34(2)$ \\
$\mathrm{N} 4-\mathrm{C} 16$ & $1.404(18)$ & $\mathrm{C} 30-\mathrm{O} 4$ & $1.50(2)$ \\
$\mathrm{N} 4-\mathrm{C} 19$ & $1.366(16)$ & $\mathrm{C} 30-\mathrm{C} 31$ & $1.54(2)$ \\
$\mathrm{C} 1-\mathrm{C} 20$ & $1.37(3)$ & $\mathrm{C} 30-\mathrm{H} 30 \mathrm{~A}$ & 0.9701 \\
$\mathrm{C} 1-\mathrm{C} 2$ & $1.39(2)$ & $\mathrm{C} 31-\mathrm{H} 30 \mathrm{~B} 31 \mathrm{~A}$ & 0.9699 \\
$\mathrm{C} 2-\mathrm{C} 3$ & $1.418(19)$ & $\mathrm{C} 31-\mathrm{H} 31 \mathrm{~B}$ & 0.9600 \\
$\mathrm{C} 2-\mathrm{C} 21$ & $1.40(3)$ & $\mathrm{C} 31-\mathrm{H} 31 \mathrm{C}$ & 0.9600 \\
$\mathrm{C} 3-\mathrm{C} 4$ & $1.43(3)$ & $\mathrm{C} 32-\mathrm{H} 32 \mathrm{~A}$ & 0.9600 \\
$\mathrm{C} 3-\mathrm{C} 26$ & $1.39(3)$ & & 0.9600 \\
$\mathrm{C} 4-\mathrm{C} 5$ & $1.513(18)$ & & 0.9600
\end{tabular}




\begin{tabular}{|c|c|c|c|}
\hline $\mathrm{C} 5-\mathrm{C} 6$ & $1.37(2)$ & $\mathrm{C} 32-\mathrm{H} 32 \mathrm{C}$ & 0.9603 \\
\hline $\mathrm{C} 5-\mathrm{H} 5$ & 0.9300 & C33-C34 & $1.46(3)$ \\
\hline $\mathrm{C} 6-\mathrm{C} 7$ & $1.45(3)$ & $\mathrm{C} 33-\mathrm{H} 33 \mathrm{~A}$ & 0.9699 \\
\hline $\mathrm{C} 7-\mathrm{C} 8$ & $1.33(2)$ & C $33-\mathrm{H} 33 \mathrm{~B}$ & 0.9700 \\
\hline $\mathrm{C} 7-\mathrm{C} 27$ & $1.50(2)$ & $\mathrm{C} 34-\mathrm{C} 35$ & $1.558(16)$ \\
\hline $\mathrm{C} 8-\mathrm{C} 9$ & $1.45(2)$ & $\mathrm{C} 34-\mathrm{H} 34 \mathrm{~A}$ & 0.9702 \\
\hline $\mathrm{C} 8-\mathrm{C} 32$ & $1.49(3)$ & С $34-\mathrm{H} 34 \mathrm{~B}$ & 0.9700 \\
\hline $\mathrm{C} 9-\mathrm{C} 10$ & $1.380(19)$ & $\mathrm{C} 35-\mathrm{O} 5$ & $1.143(17)$ \\
\hline $\mathrm{C} 10-\mathrm{C} 11$ & $1.39(2)$ & $\mathrm{C} 35-\mathrm{O} 6$ & $1.35(2)$ \\
\hline $\mathrm{C} 10-\mathrm{H} 10$ & 0.9299 & $\mathrm{C} 36-\mathrm{C} 37$ & $1.49(3)$ \\
\hline $\mathrm{C} 11-\mathrm{C} 12$ & 1.467 (19) & $\mathrm{C} 36-\mathrm{O} 6$ & $1.512(19)$ \\
\hline $\mathrm{C} 12-\mathrm{C} 13$ & $1.36(3)$ & $\mathrm{C} 36-\mathrm{H} 36 \mathrm{~A}$ & 0.9700 \\
\hline $\mathrm{C} 12-\mathrm{C} 33$ & $1.45(3)$ & C36-H36B & 0.9701 \\
\hline $\mathrm{C} 13-\mathrm{C} 14$ & $1.44(3)$ & C37-H37A & 0.9601 \\
\hline $\mathrm{C} 13-\mathrm{C} 38$ & $1.512(17)$ & C37-H37B & 0.9599 \\
\hline $\mathrm{C} 14-\mathrm{C} 15$ & $1.37(3)$ & C37-H37C & 0.9602 \\
\hline $\mathrm{C} 15-\mathrm{C} 16$ & $1.40(3)$ & C $38-\mathrm{H} 38 \mathrm{~A}$ & 0.9600 \\
\hline $\mathrm{C} 15-\mathrm{H} 15$ & 0.9300 & C $38-\mathrm{H} 38 \mathrm{~B}$ & 0.9599 \\
\hline $\mathrm{C} 16-\mathrm{C} 17$ & $1.44(3)$ & $\mathrm{C} 38-\mathrm{H} 38 \mathrm{C}$ & 0.9601 \\
\hline $\mathrm{C} 17-\mathrm{C} 18$ & $1.37(2)$ & $\mathrm{C} 39-\mathrm{C} 40$ & $1.54(2)$ \\
\hline $\mathrm{C} 17-\mathrm{C} 39$ & $1.55(2)$ & C39-H39A & 0.9701 \\
\hline $\mathrm{C} 18-\mathrm{C} 19$ & $1.45(3)$ & C39-H39B & 0.9699 \\
\hline $\mathrm{C} 18-\mathrm{C} 44$ & $1.52(3)$ & $\mathrm{C} 40-\mathrm{C} 41$ & $1.49(2)$ \\
\hline $\mathrm{C} 19-\mathrm{C} 20$ & $1.385(18)$ & $\mathrm{C} 40-\mathrm{H} 40 \mathrm{~A}$ & 0.9701 \\
\hline $\mathrm{C} 20-\mathrm{H} 20$ & 0.9299 & $\mathrm{C} 40-\mathrm{H} 40 \mathrm{~B}$ & 0.9699 \\
\hline $\mathrm{C} 21-\mathrm{C} 22$ & $1.45(3)$ & $\mathrm{C} 41-\mathrm{O} 7$ & $1.21(2)$ \\
\hline $\mathrm{C} 21-\mathrm{H} 21 \mathrm{~A}$ & 0.9699 & $\mathrm{C} 41-\mathrm{O} 8$ & $1.297(18)$ \\
\hline $\mathrm{C} 21-\mathrm{H} 21 \mathrm{~B}$ & 0.9701 & $\mathrm{C} 42-\mathrm{C} 43$ & $1.48(2)$ \\
\hline $\mathrm{C} 22-\mathrm{C} 23$ & $1.55(3)$ & $\mathrm{C} 42-\mathrm{O} 8$ & $1.48(2)$ \\
\hline $\mathrm{C} 22-\mathrm{H} 22 \mathrm{~A}$ & 0.9700 & $\mathrm{C} 42-\mathrm{H} 42 \mathrm{~A}$ & 0.9700 \\
\hline $\mathrm{C} 22-\mathrm{H} 22 \mathrm{~B}$ & 0.9700 & $\mathrm{C} 42-\mathrm{H} 42 \mathrm{~B}$ & 0.9699 \\
\hline $\mathrm{C} 23-\mathrm{O} 1$ & $1.16(2)$ & $\mathrm{C} 43-\mathrm{H} 43 \mathrm{~A}$ & 0.9601 \\
\hline $\mathrm{C} 23-\mathrm{O} 2$ & $1.36(2)$ & $\mathrm{C} 43-\mathrm{H} 43 \mathrm{~B}$ & 0.9599 \\
\hline $\mathrm{C} 24-\mathrm{O} 2$ & $1.44(3)$ & $\mathrm{C} 43-\mathrm{H} 43 \mathrm{C}$ & 0.9600 \\
\hline $\mathrm{C} 24-\mathrm{C} 25$ & $1.50(3)$ & $\mathrm{C} 44-\mathrm{H} 44 \mathrm{~A}$ & 0.9600 \\
\hline $\mathrm{C} 24-\mathrm{H} 24 \mathrm{~A}$ & 0.9700 & C44-H44B & 0.9600 \\
\hline $\mathrm{C} 24-\mathrm{H} 24 \mathrm{~B}$ & 0.9700 & $\mathrm{C} 44-\mathrm{H} 44 \mathrm{C}$ & 0.9600 \\
\hline $\mathrm{C} 25-\mathrm{H} 25 \mathrm{~A}$ & 0.9601 & $\mathrm{O} 5-\mathrm{Ru} 1^{\mathrm{ii}}$ & $2.194(11)$ \\
\hline $\mathrm{C} 25-\mathrm{H} 25 \mathrm{~B}$ & 0.9599 & $\mathrm{O} 9-\mathrm{C} 45$ & $1.17(2)$ \\
\hline $\mathrm{C} 45-\mathrm{Ru} 1-\mathrm{N} 2$ & $95.7(7)$ & $\mathrm{H} 25 \mathrm{~B}-\mathrm{C} 25-\mathrm{H} 25 \mathrm{C}$ & 109.5 \\
\hline $\mathrm{C} 45-\mathrm{Ru} 1-\mathrm{N} 4$ & $90.2(7)$ & $\mathrm{C} 3-\mathrm{C} 26-\mathrm{H} 26 \mathrm{~A}$ & 109.5 \\
\hline N2-Ru1-N4 & $173.8(5)$ & $\mathrm{C} 3-\mathrm{C} 26-\mathrm{H} 26 \mathrm{~B}$ & 109.5 \\
\hline $\mathrm{C} 45-\mathrm{Ru} 1-\mathrm{N} 1$ & $95.9(7)$ & $\mathrm{H} 26 \mathrm{~A}-\mathrm{C} 26-\mathrm{H} 26 \mathrm{~B}$ & 109.5 \\
\hline N2-Ru1-N1 & $91.7(6)$ & $\mathrm{C} 3-\mathrm{C} 26-\mathrm{H} 26 \mathrm{C}$ & 109.5 \\
\hline N4-Ru1-N1 & $89.3(5)$ & $\mathrm{H} 26 \mathrm{~A}-\mathrm{C} 26-\mathrm{H} 26 \mathrm{C}$ & 109.5 \\
\hline $\mathrm{C} 45-\mathrm{Ru} 1-\mathrm{N} 3$ & $89.6(7)$ & $\mathrm{H} 26 \mathrm{~B}-\mathrm{C} 26-\mathrm{H} 26 \mathrm{C}$ & 109.5 \\
\hline $\mathrm{N} 2-\mathrm{Ru} 1-\mathrm{N} 3$ & $87.9(6)$ & $\mathrm{C} 7-\mathrm{C} 27-\mathrm{C} 28$ & $111.4(12)$ \\
\hline
\end{tabular}




\begin{tabular}{|c|c|c|c|}
\hline $\mathrm{N} 4-\mathrm{Ru} 1-\mathrm{N} 3$ & $90.5(5)$ & $\mathrm{C} 7-\mathrm{C} 27-\mathrm{H} 27 \mathrm{~A}$ & 109.3 \\
\hline $\mathrm{N} 1-\mathrm{Ru} 1-\mathrm{N} 3$ & $174.5(5)$ & $\mathrm{C} 28-\mathrm{C} 27-\mathrm{H} 27 \mathrm{~A}$ & 109.3 \\
\hline $\mathrm{C} 45-\mathrm{Ru} 1-\mathrm{O}^{\mathrm{i}}$ & $175.2(8)$ & $\mathrm{C} 7-\mathrm{C} 27-\mathrm{H} 27 \mathrm{~B}$ & 109.3 \\
\hline $\mathrm{N} 2-\mathrm{Ru} 1-\mathrm{O}^{\mathrm{i}}$ & $87.0(5)$ & $\mathrm{C} 28-\mathrm{C} 27-\mathrm{H} 27 \mathrm{~B}$ & 109.3 \\
\hline $\mathrm{N} 4-\mathrm{Ru} 1-\mathrm{O} 5^{\mathrm{i}}$ & $87.1(5)$ & $\mathrm{H} 27 \mathrm{~A}-\mathrm{C} 27-\mathrm{H} 27 \mathrm{~B}$ & 108.0 \\
\hline $\mathrm{N} 1-\mathrm{Ru} 1-\mathrm{O} 5^{\mathrm{i}}$ & $80.0(5)$ & $\mathrm{C} 29-\mathrm{C} 28-\mathrm{C} 27$ & $117.0(15)$ \\
\hline $\mathrm{N} 3-\mathrm{Ru} 1-\mathrm{O} 5^{\mathrm{i}}$ & $94.4(5)$ & $\mathrm{C} 29-\mathrm{C} 28-\mathrm{H} 28 \mathrm{~A}$ & 108.1 \\
\hline $\mathrm{C} 1-\mathrm{N} 1-\mathrm{C} 4$ & $104.8(12)$ & $\mathrm{C} 27-\mathrm{C} 28-\mathrm{H} 28 \mathrm{~A}$ & 108.0 \\
\hline $\mathrm{C} 1-\mathrm{N} 1-\mathrm{Ru} 1$ & $128.7(10)$ & $\mathrm{C} 29-\mathrm{C} 28-\mathrm{H} 28 \mathrm{~B}$ & 108.1 \\
\hline $\mathrm{C} 4-\mathrm{N} 1-\mathrm{Ru} 1$ & $126.3(13)$ & $\mathrm{C} 27-\mathrm{C} 28-\mathrm{H} 28 \mathrm{~B}$ & 108.0 \\
\hline $\mathrm{C} 6-\mathrm{N} 2-\mathrm{C} 9$ & $106.2(15)$ & $\mathrm{H} 28 \mathrm{~A}-\mathrm{C} 28-\mathrm{H} 28 \mathrm{~B}$ & 107.3 \\
\hline $\mathrm{C} 6-\mathrm{N} 2-\mathrm{Ru} 1$ & $124.7(14)$ & $\mathrm{O} 3-\mathrm{C} 29-\mathrm{O} 4$ & $117.5(16)$ \\
\hline $\mathrm{C} 9-\mathrm{N} 2-\mathrm{Ru} 1$ & $128.9(9)$ & $\mathrm{O} 3-\mathrm{C} 29-\mathrm{C} 28$ & $121.8(16)$ \\
\hline $\mathrm{C} 11-\mathrm{N} 3-\mathrm{C} 14$ & $106.1(13)$ & $\mathrm{O} 4-\mathrm{C} 29-\mathrm{C} 28$ & $120.6(17)$ \\
\hline $\mathrm{C} 11-\mathrm{N} 3-\mathrm{Ru} 1$ & $127.2(9)$ & $\mathrm{O} 4-\mathrm{C} 30-\mathrm{C} 31$ & $106.7(17)$ \\
\hline $\mathrm{C} 14-\mathrm{N} 3-\mathrm{Ru} 1$ & $126.6(13)$ & $\mathrm{O} 4-\mathrm{C} 30-\mathrm{H} 30 \mathrm{~A}$ & 110.4 \\
\hline $\mathrm{C} 16-\mathrm{N} 4-\mathrm{C} 19$ & $107.1(15)$ & $\mathrm{C} 31-\mathrm{C} 30-\mathrm{H} 30 \mathrm{~A}$ & 110.4 \\
\hline $\mathrm{C} 16-\mathrm{N} 4-\mathrm{Ru} 1$ & $126.2(13)$ & $\mathrm{O} 4-\mathrm{C} 30-\mathrm{H} 30 \mathrm{~B}$ & 110.4 \\
\hline $\mathrm{C} 19-\mathrm{N} 4-\mathrm{Ru} 1$ & $126.6(8)$ & $\mathrm{C} 31-\mathrm{C} 30-\mathrm{H} 30 \mathrm{~B}$ & 110.4 \\
\hline $\mathrm{N} 1-\mathrm{C} 1-\mathrm{C} 20$ & $121.7(13)$ & $\mathrm{H} 30 \mathrm{~A}-\mathrm{C} 30-\mathrm{H} 30 \mathrm{~B}$ & 108.6 \\
\hline $\mathrm{N} 1-\mathrm{C} 1-\mathrm{C} 2$ & $110.5(15)$ & $\mathrm{C} 30-\mathrm{C} 31-\mathrm{H} 31 \mathrm{~A}$ & 109.5 \\
\hline $\mathrm{C} 20-\mathrm{C} 1-\mathrm{C} 2$ & $127.7(19)$ & $\mathrm{C} 30-\mathrm{C} 31-\mathrm{H} 31 \mathrm{~B}$ & 109.5 \\
\hline $\mathrm{C} 3-\mathrm{C} 2-\mathrm{C} 1$ & $106.7(17)$ & $\mathrm{H} 31 \mathrm{~A}-\mathrm{C} 31-\mathrm{H} 31 \mathrm{~B}$ & 109.5 \\
\hline $\mathrm{C} 3-\mathrm{C} 2-\mathrm{C} 21$ & $120.6(13)$ & $\mathrm{C} 30-\mathrm{C} 31-\mathrm{H} 31 \mathrm{C}$ & 109.5 \\
\hline $\mathrm{C} 1-\mathrm{C} 2-\mathrm{C} 21$ & $132.8(18)$ & $\mathrm{H} 31 \mathrm{~A}-\mathrm{C} 31-\mathrm{H} 31 \mathrm{C}$ & 109.5 \\
\hline $\mathrm{C} 4-\mathrm{C} 3-\mathrm{C} 2$ & $107.7(13)$ & $\mathrm{H} 31 \mathrm{~B}-\mathrm{C} 31-\mathrm{H} 31 \mathrm{C}$ & 109.5 \\
\hline $\mathrm{C} 4-\mathrm{C} 3-\mathrm{C} 26$ & 130.7 (19) & $\mathrm{C} 8-\mathrm{C} 32-\mathrm{H} 32 \mathrm{~A}$ & 109.5 \\
\hline $\mathrm{C} 2-\mathrm{C} 3-\mathrm{C} 26$ & $121.5(17)$ & $\mathrm{C} 8-\mathrm{C} 32-\mathrm{H} 32 \mathrm{~B}$ & 109.5 \\
\hline $\mathrm{C} 5-\mathrm{C} 4-\mathrm{C} 3$ & $128.9(13)$ & $\mathrm{H} 32 \mathrm{~A}-\mathrm{C} 32-\mathrm{H} 32 \mathrm{~B}$ & 109.5 \\
\hline $\mathrm{C} 5-\mathrm{C} 4-\mathrm{N} 1$ & $120.8(14)$ & $\mathrm{C} 8-\mathrm{C} 32-\mathrm{H} 32 \mathrm{C}$ & 109.5 \\
\hline $\mathrm{C} 3-\mathrm{C} 4-\mathrm{N} 1$ & $110.2(17)$ & $\mathrm{H} 32 \mathrm{~A}-\mathrm{C} 32-\mathrm{H} 32 \mathrm{C}$ & 109.4 \\
\hline $\mathrm{C} 6-\mathrm{C} 5-\mathrm{C} 4$ & $131.0(13)$ & $\mathrm{H} 32 \mathrm{~B}-\mathrm{C} 32-\mathrm{H} 32 \mathrm{C}$ & 109.4 \\
\hline $\mathrm{C} 6-\mathrm{C} 5-\mathrm{H} 5$ & 114.5 & $\mathrm{C} 12-\mathrm{C} 33-\mathrm{C} 34$ & $120.2(18)$ \\
\hline $\mathrm{C} 4-\mathrm{C} 5-\mathrm{H} 5$ & 114.5 & $\mathrm{C} 12-\mathrm{C} 33-\mathrm{H} 33 \mathrm{~A}$ & 107.3 \\
\hline $\mathrm{C} 5-\mathrm{C} 6-\mathrm{N} 2$ & $125.2(18)$ & $\mathrm{C} 34-\mathrm{C} 33-\mathrm{H} 33 \mathrm{~A}$ & 107.3 \\
\hline $\mathrm{C} 5-\mathrm{C} 6-\mathrm{C} 7$ & $125.9(13)$ & $\mathrm{C} 12-\mathrm{C} 33-\mathrm{H} 33 \mathrm{~B}$ & 107.3 \\
\hline $\mathrm{N} 2-\mathrm{C} 6-\mathrm{C} 7$ & $108.9(15)$ & C $34-\mathrm{C} 33-\mathrm{H} 33 \mathrm{~B}$ & 107.3 \\
\hline $\mathrm{C} 8-\mathrm{C} 7-\mathrm{C} 6$ & $108.2(15)$ & $\mathrm{H} 33 \mathrm{~A}-\mathrm{C} 33-\mathrm{H} 33 \mathrm{~B}$ & 106.9 \\
\hline $\mathrm{C} 8-\mathrm{C} 7-\mathrm{C} 27$ & $129(2)$ & $\mathrm{C} 33-\mathrm{C} 34-\mathrm{C} 35$ & $109.8(15)$ \\
\hline $\mathrm{C} 6-\mathrm{C} 7-\mathrm{C} 27$ & $123.2(15)$ & $\mathrm{C} 33-\mathrm{C} 34-\mathrm{H} 34 \mathrm{~A}$ & 109.7 \\
\hline $\mathrm{C} 7-\mathrm{C} 8-\mathrm{C} 9$ & $107.2(19)$ & $\mathrm{C} 35-\mathrm{C} 34-\mathrm{H} 34 \mathrm{~A}$ & 109.7 \\
\hline $\mathrm{C} 7-\mathrm{C} 8-\mathrm{C} 32$ & $129.6(16)$ & $\mathrm{C} 33-\mathrm{C} 34-\mathrm{H} 34 \mathrm{~B}$ & 109.7 \\
\hline $\mathrm{C} 9-\mathrm{C} 8-\mathrm{C} 32$ & $123.2(13)$ & C35- C34-H34B & 109.7 \\
\hline $\mathrm{C} 10-\mathrm{C} 9-\mathrm{N} 2$ & $123.8(16)$ & $\mathrm{H} 34 \mathrm{~A}-\mathrm{C} 34-\mathrm{H} 34 \mathrm{~B}$ & 108.2 \\
\hline $\mathrm{C} 10-\mathrm{C} 9-\mathrm{C} 8$ & $126.7(18)$ & $\mathrm{O} 5-\mathrm{C} 35-\mathrm{O} 6$ & $125.9(11)$ \\
\hline $\mathrm{N} 2-\mathrm{C} 9-\mathrm{C} 8$ & $109.4(13)$ & $\mathrm{O} 5-\mathrm{C} 35-\mathrm{C} 34$ & $118.1(14)$ \\
\hline $\mathrm{C} 9-\mathrm{C} 10-\mathrm{C} 11$ & $125.3(19)$ & $\mathrm{O} 6-\mathrm{C} 35-\mathrm{C} 34$ & $115.4(11)$ \\
\hline $\mathrm{C} 9-\mathrm{C} 10-\mathrm{H} 10$ & 117.4 & $\mathrm{C} 37-\mathrm{C} 36-\mathrm{O} 6$ & $107.1(17)$ \\
\hline
\end{tabular}




\begin{tabular}{|c|c|}
\hline $\mathrm{C} 11-\mathrm{C} 10-\mathrm{H} 10$ & 117.4 \\
\hline $\mathrm{N} 3-\mathrm{C} 11-\mathrm{C} 10$ & $126.5(14)$ \\
\hline $\mathrm{N} 3-\mathrm{C} 11-\mathrm{C} 12$ & $111.3(14)$ \\
\hline $\mathrm{C} 10-\mathrm{C} 11-\mathrm{C} 12$ & $122.1(18)$ \\
\hline $\mathrm{C} 13-\mathrm{C} 12-\mathrm{C} 33$ & $131.7(13)$ \\
\hline $\mathrm{C} 13-\mathrm{C} 12-\mathrm{C} 11$ & $105.5(16)$ \\
\hline $\mathrm{C} 33-\mathrm{C} 12-\mathrm{C} 11$ & $122.6(15)$ \\
\hline $\mathrm{C} 12-\mathrm{C} 13-\mathrm{C} 14$ & $108.1(13)$ \\
\hline $\mathrm{C} 12-\mathrm{C} 13-\mathrm{C} 38$ & $119.2(17)$ \\
\hline $\mathrm{C} 14-\mathrm{C} 13-\mathrm{C} 38$ & $132.5(17)$ \\
\hline $\mathrm{C} 15-\mathrm{C} 14-\mathrm{N} 3$ & $122.1(15)$ \\
\hline $\mathrm{C} 15-\mathrm{C} 14-\mathrm{C} 13$ & $128.9(13)$ \\
\hline $\mathrm{N} 3-\mathrm{C} 14-\mathrm{C} 13$ & $109.0(17)$ \\
\hline $\mathrm{C} 14-\mathrm{C} 15-\mathrm{C} 16$ & $129.7(13)$ \\
\hline $\mathrm{C} 14-\mathrm{C} 15-\mathrm{H} 15$ & 115.2 \\
\hline $\mathrm{C} 16-\mathrm{C} 15-\mathrm{H} 15$ & 115.2 \\
\hline $\mathrm{N} 4-\mathrm{C} 16-\mathrm{C} 15$ & $124.7(18)$ \\
\hline $\mathrm{N} 4-\mathrm{C} 16-\mathrm{C} 17$ & $109.5(15)$ \\
\hline $\mathrm{C} 15-\mathrm{C} 16-\mathrm{C} 17$ & $125.7(13)$ \\
\hline $\mathrm{C} 18-\mathrm{C} 17-\mathrm{C} 16$ & $107.7(14)$ \\
\hline $\mathrm{C} 18-\mathrm{C} 17-\mathrm{C} 39$ & $124.2(19)$ \\
\hline $\mathrm{C} 16-\mathrm{C} 17-\mathrm{C} 39$ & $128.0(15)$ \\
\hline $\mathrm{C} 17-\mathrm{C} 18-\mathrm{C} 19$ & $105.7(18)$ \\
\hline $\mathrm{C} 17-\mathrm{C} 18-\mathrm{C} 44$ & $119.3(15)$ \\
\hline $\mathrm{C} 19-\mathrm{C} 18-\mathrm{C} 44$ & $134.9(13)$ \\
\hline $\mathrm{N} 4-\mathrm{C} 19-\mathrm{C} 20$ & $125.4(17)$ \\
\hline $\mathrm{N} 4-\mathrm{C} 19-\mathrm{C} 18$ & $109.8(12)$ \\
\hline $\mathrm{C} 20-\mathrm{C} 19-\mathrm{C} 18$ & 124.7 (19) \\
\hline $\mathrm{C} 19-\mathrm{C} 20-\mathrm{C} 1$ & $128.0(19)$ \\
\hline $\mathrm{C} 19-\mathrm{C} 20-\mathrm{H} 20$ & 116.0 \\
\hline $\mathrm{C} 1-\mathrm{C} 20-\mathrm{H} 20$ & 116.0 \\
\hline $\mathrm{C} 2-\mathrm{C} 21-\mathrm{C} 22$ & $122.4(11)$ \\
\hline $\mathrm{C} 2-\mathrm{C} 21-\mathrm{H} 21 \mathrm{~A}$ & 106.7 \\
\hline $\mathrm{C} 22-\mathrm{C} 21-\mathrm{H} 21 \mathrm{~A}$ & 106.7 \\
\hline $\mathrm{C} 2-\mathrm{C} 21-\mathrm{H} 21 \mathrm{~B}$ & 106.7 \\
\hline $\mathrm{C} 22-\mathrm{C} 21-\mathrm{H} 21 \mathrm{~B}$ & 106.7 \\
\hline $\mathrm{H} 21 \mathrm{~A}-\mathrm{C} 21-\mathrm{H} 21 \mathrm{~B}$ & 106.6 \\
\hline $\mathrm{C} 21-\mathrm{C} 22-\mathrm{C} 23$ & $112.8(11)$ \\
\hline $\mathrm{C} 21-\mathrm{C} 22-\mathrm{H} 22 \mathrm{~A}$ & 109.0 \\
\hline $\mathrm{C} 23-\mathrm{C} 22-\mathrm{H} 22 \mathrm{~A}$ & 109.0 \\
\hline $\mathrm{C} 21-\mathrm{C} 22-\mathrm{H} 22 \mathrm{~B}$ & 109.0 \\
\hline $\mathrm{C} 23-\mathrm{C} 22-\mathrm{H} 22 \mathrm{~B}$ & 109.0 \\
\hline $\mathrm{H} 22 \mathrm{~A}-\mathrm{C} 22-\mathrm{H} 22 \mathrm{~B}$ & 107.8 \\
\hline $\mathrm{O} 1-\mathrm{C} 23-\mathrm{O} 2$ & $124.3(16)$ \\
\hline $\mathrm{O} 1-\mathrm{C} 23-\mathrm{C} 22$ & $119.5(16)$ \\
\hline $\mathrm{O} 2-\mathrm{C} 23-\mathrm{C} 22$ & $115.4(16)$ \\
\hline $\mathrm{O} 2-\mathrm{C} 24-\mathrm{C} 25$ & $112.9(15)$ \\
\hline & \\
\hline
\end{tabular}

\begin{tabular}{|c|c|}
\hline $\mathrm{C} 37-\mathrm{C} 36-\mathrm{H} 36 \mathrm{~A}$ & 110.3 \\
\hline $\mathrm{O} 6-\mathrm{C} 36-\mathrm{H} 36 \mathrm{~A}$ & 110.3 \\
\hline $\mathrm{C} 37-\mathrm{C} 36-\mathrm{H} 36 \mathrm{~B}$ & 110.3 \\
\hline $\mathrm{O} 6-\mathrm{C} 36-\mathrm{H} 36 \mathrm{~B}$ & 110.3 \\
\hline $\mathrm{H} 36 \mathrm{~A}-\mathrm{C} 36-\mathrm{H} 36 \mathrm{~B}$ & 108.5 \\
\hline $\mathrm{C} 36-\mathrm{C} 37-\mathrm{H} 37 \mathrm{~A}$ & 109.5 \\
\hline $\mathrm{C} 36-\mathrm{C} 37-\mathrm{H} 37 \mathrm{~B}$ & 109.5 \\
\hline $\mathrm{H} 37 \mathrm{~A}-\mathrm{C} 37-\mathrm{H} 37 \mathrm{~B}$ & 109.5 \\
\hline C36-C37-H37C & 109.5 \\
\hline $\mathrm{H} 37 \mathrm{~A}-\mathrm{C} 37-\mathrm{H} 37 \mathrm{C}$ & 109.5 \\
\hline H37B-C37-H37C & 109.5 \\
\hline $\mathrm{C} 13-\mathrm{C} 38-\mathrm{H} 38 \mathrm{~A}$ & 109.5 \\
\hline $\mathrm{C} 13-\mathrm{C} 38-\mathrm{H} 38 \mathrm{~B}$ & 109.5 \\
\hline $\mathrm{H} 38 \mathrm{~A}-\mathrm{C} 38-\mathrm{H} 38 \mathrm{~B}$ & 109.5 \\
\hline C13-C38-H38C & 109.5 \\
\hline $\mathrm{H} 38 \mathrm{~A}-\mathrm{C} 38-\mathrm{H} 38 \mathrm{C}$ & 109.5 \\
\hline $\mathrm{H} 38 \mathrm{~B}-\mathrm{C} 38-\mathrm{H} 38 \mathrm{C}$ & 109.5 \\
\hline $\mathrm{C} 40-\mathrm{C} 39-\mathrm{C} 17$ & $108.2(14)$ \\
\hline $\mathrm{C} 40-\mathrm{C} 39-\mathrm{H} 39 \mathrm{~A}$ & 110.1 \\
\hline $\mathrm{C} 17-\mathrm{C} 39-\mathrm{H} 39 \mathrm{~A}$ & 110.1 \\
\hline $\mathrm{C} 40-\mathrm{C} 39-\mathrm{H} 39 \mathrm{~B}$ & 110.1 \\
\hline С17- $339-\mathrm{H} 39 \mathrm{~B}$ & 110.1 \\
\hline $\mathrm{H} 39 \mathrm{~A}-\mathrm{C} 39-\mathrm{H} 39 \mathrm{~B}$ & 108.4 \\
\hline $\mathrm{C} 41-\mathrm{C} 40-\mathrm{C} 39$ & $113.1(15)$ \\
\hline $\mathrm{C} 41-\mathrm{C} 40-\mathrm{H} 40 \mathrm{~A}$ & 109.0 \\
\hline $\mathrm{C} 39-\mathrm{C} 40-\mathrm{H} 40 \mathrm{~A}$ & 109.0 \\
\hline $\mathrm{C} 41-\mathrm{C} 40-\mathrm{H} 40 \mathrm{~B}$ & 109.0 \\
\hline $\mathrm{C} 39-\mathrm{C} 40-\mathrm{H} 40 \mathrm{~B}$ & 109.0 \\
\hline $\mathrm{H} 40 \mathrm{~A}-\mathrm{C} 40-\mathrm{H} 40 \mathrm{~B}$ & 107.8 \\
\hline $\mathrm{O} 7-\mathrm{C} 41-\mathrm{O} 8$ & $119.2(16)$ \\
\hline $\mathrm{O} 7-\mathrm{C} 41-\mathrm{C} 40$ & $115.9(13)$ \\
\hline $\mathrm{O} 8-\mathrm{C} 41-\mathrm{C} 40$ & $124.6(16)$ \\
\hline $\mathrm{C} 43-\mathrm{C} 42-\mathrm{O} 8$ & $111.1(17)$ \\
\hline $\mathrm{C} 43-\mathrm{C} 42-\mathrm{H} 42 \mathrm{~A}$ & 109.4 \\
\hline $\mathrm{O} 8-\mathrm{C} 42-\mathrm{H} 42 \mathrm{~A}$ & 109.4 \\
\hline $\mathrm{C} 43-\mathrm{C} 42-\mathrm{H} 42 \mathrm{~B}$ & 109.4 \\
\hline $\mathrm{O} 8-\mathrm{C} 42-\mathrm{H} 42 \mathrm{~B}$ & 109.4 \\
\hline $\mathrm{H} 42 \mathrm{~A}-\mathrm{C} 42-\mathrm{H} 42 \mathrm{~B}$ & 108.0 \\
\hline $\mathrm{C} 42-\mathrm{C} 43-\mathrm{H} 43 \mathrm{~A}$ & 109.5 \\
\hline $\mathrm{C} 42-\mathrm{C} 43-\mathrm{H} 43 \mathrm{~B}$ & 109.5 \\
\hline $\mathrm{H} 43 \mathrm{~A}-\mathrm{C} 43-\mathrm{H} 43 \mathrm{~B}$ & 109.5 \\
\hline $\mathrm{C} 42-\mathrm{C} 43-\mathrm{H} 43 \mathrm{C}$ & 109.5 \\
\hline $\mathrm{H} 43 \mathrm{~A}-\mathrm{C} 43-\mathrm{H} 43 \mathrm{C}$ & 109.5 \\
\hline $\mathrm{H} 43 \mathrm{~B}-\mathrm{C} 43-\mathrm{H} 43 \mathrm{C}$ & 109.5 \\
\hline $\mathrm{C} 18-\mathrm{C} 44-\mathrm{H} 44 \mathrm{~A}$ & 109.5 \\
\hline $\mathrm{C} 18-\mathrm{C} 44-\mathrm{H} 44 \mathrm{~B}$ & 109.5 \\
\hline $\mathrm{H} 44 \mathrm{~A}-\mathrm{C} 44-\mathrm{H} 44 \mathrm{~B}$ & 109.5 \\
\hline $\mathrm{C} 18-\mathrm{C} 44-\mathrm{H} 44 \mathrm{C}$ & 109.5 \\
\hline
\end{tabular}


supporting information

$\begin{array}{llll}\mathrm{C} 25-\mathrm{C} 24-\mathrm{H} 24 \mathrm{~A} & 109.0 & \mathrm{H} 44 \mathrm{~A}-\mathrm{C} 44-\mathrm{H} 44 \mathrm{C} & 109.5 \\ \mathrm{O} 2-\mathrm{C} 24-\mathrm{H} 24 \mathrm{~B} & 109.0 & \mathrm{H} 44 \mathrm{~B}-\mathrm{C} 44-\mathrm{H} 44 \mathrm{C} & 109.5 \\ \mathrm{C} 25-\mathrm{C} 24-\mathrm{H} 24 \mathrm{~B} & 109.0 & \mathrm{C} 23-\mathrm{O} 2-\mathrm{C} 24 & 127.3(17) \\ \mathrm{H} 24 \mathrm{~A}-\mathrm{C} 24-\mathrm{H} 24 \mathrm{~B} & 107.8 & \mathrm{C} 29-\mathrm{O} 4-\mathrm{C} 30 & 125.7(15) \\ \mathrm{C} 24-\mathrm{C} 25-\mathrm{H} 25 \mathrm{~A} & 109.5 & \mathrm{C} 35-\mathrm{O} 5-\mathrm{Ru} 1^{\mathrm{ii}} & 162.6(10) \\ \mathrm{C} 24-\mathrm{C} 25-\mathrm{H} 25 \mathrm{~B} & 109.5 & \mathrm{C} 35-\mathrm{O} 6-\mathrm{C} 36 & 115.1(12) \\ \mathrm{H} 25 \mathrm{~A}-\mathrm{C} 25-\mathrm{H} 25 \mathrm{~B} & 109.5 & \mathrm{C} 41-\mathrm{O} 8-\mathrm{C} 42 & 127.0(14) \\ \mathrm{C} 24-\mathrm{C} 25-\mathrm{H} 25 \mathrm{C} & 109.5 & \mathrm{O} 9-\mathrm{C} 45-\mathrm{Ru} 1 & 173.9(13) \\ \mathrm{H} 25 \mathrm{~A}-\mathrm{C} 25-\mathrm{H} 25 \mathrm{C} & 109.5 & & \end{array}$

Symmetry codes: (i) $-x+3 / 2, y-1 / 2,-z+3 / 2$; (ii) $-x+3 / 2, y+1 / 2,-z+3 / 2$.

Hydrogen-bond geometry $\left(A,{ }^{\circ}\right)$

\begin{tabular}{lllll}
\hline$D-\mathrm{H} \cdots A$ & $D-\mathrm{H}$ & $\mathrm{H} \cdots A$ & $D \cdots A$ & $D-\mathrm{H} \cdots A$ \\
\hline $\mathrm{C} 25-\mathrm{H} 25 C \cdots \mathrm{O} 2^{\mathrm{iii}}$ & 0.96 & 2.17 & $3.08(2)$ & 157 \\
$\mathrm{C} 37-\mathrm{H} 37 C \cdots \mathrm{O} 9^{\mathrm{i}}$ & 0.96 & 2.29 & $2.93(2)$ & 123 \\
$\mathrm{C} 39-\mathrm{H} 39 A \cdots 7^{\text {iv }}$ & 0.97 & 2.43 & $3.37(2)$ & 161 \\
$\mathrm{C} 39-\mathrm{H} 39 B \cdots{ }^{\mathrm{O}}$ & 0.97 & 2.54 & $3.51(2)$ & 173 \\
$\mathrm{C} 43-\mathrm{H} 43 B \cdots{ }^{\mathrm{v}}$ & 0.96 & 2.19 & $3.00(2)$ & 141 \\
$\mathrm{C} 44-\mathrm{H} 44 A \cdots \mathrm{O} 1^{\mathrm{iii}}$ & 0.96 & 2.60 & $3.29(2)$ & 129
\end{tabular}

Symmetry codes: (i) $-x+3 / 2, y-1 / 2,-z+3 / 2$; (iii) $-x+2, y,-z+3 / 2$; (iv) $-x+3 / 2,-y+3 / 2,-z+1$; (v) $x,-y+1, z-1 / 2$; (vi) $x-1 / 2,-y+1 / 2, z-1 / 2$. 